\title{
A Core-shell Nanosensor Used for Localized Surface Plasmon Resonances Sensing
}

\author{
Shaobo Yang ${ }^{1, \text { a }}$, Wanrong $\mathrm{Liu}^{2, \mathrm{~b}}$, Xingfei $\mathrm{Li}^{1^{*}, \mathrm{c}}$ and Wenbin $\mathrm{Tan}^{3, \mathrm{~d}}$ \\ ${ }^{1}$ State Key Laboratory of Precision Measuring Technology and Instruments, Tianjin University, \\ Tianjin 300072, China; \\ ${ }^{2}$ The First Affiliated Hospital of Tianjin University of Traditional Chinese Medicine, Tianjin 300072 , \\ China. \\ ${ }^{3}$ School of Mechanical Engineering, Tianjin University of Commerce, Tianjin 300134, China \\ ayangskyle@tju.edu.cn, ${ }^{\text {b } 785736715 @ q q . c o m, ~}{ }^{c}$ lixf@tju.edu.cn, ${ }^{d}$ twb@tju.edu.cn
}

Keywords: Core-shell, Nanosensor, LSPR.

\begin{abstract}
A core-shell nanosensor was fabricated by molecular self-assemble and evaporation technique in this paper. The optical property of that was measured based on the detection system of LSPR. The sensitivity of this core-shell nanosensor is $300 \mathrm{~nm} / \mathrm{RIU}$ and the decay length is $26 \mathrm{~nm}$ based LSPR detection system. The results show that this nansensor can reach high sensitivity and be applied for the small molecule or low concentration detection.
\end{abstract}

\section{Introduction}

Noble nanoparticles such as gold and silver have attracted much attention owing to unique optical properties in biosensor during recent years [1-3]. One of the most interesting properties known as localized surface plasmon resonances (LSPR) which comes from the collective oscillation of the free electrons exited by incident electromagnetic wave [4-5]. It is reported that the properties of nanoparticles are dependent on the shape, size and composition of the nanostructures [6]. The position of plasmon resonances is excessively sensitive to the tiny variety of the local environment of the nanoparticles [7]. Therefore, the sensors based on LSPR sensing are suitable for the molecular detection with low concentration, especially in chemical and biological sensing.

The LSPR sensing principle mainly depends on the spectral shifts caused by the surrounding dielectric environmental change when adsorbents bind to the surface of the nanoparticle. The optical responses of LSPR sensing are determined not only on the refractive index of the medium but also on the distance from the metal surface.. The decay depends on the type of metal and physical parameters of the nanoparticles can be best described via the following equation [8]:

$$
R=m \Delta n[1-\exp (-d / 1)(1)
$$

where $\mathrm{R}$ is the wave-length shift or intensity change; $\mathrm{m}$ is the refractive index sensitivity, that is, resonant peak shift or intensity change per refractive index unit (RIU) change; $\Delta \mathrm{n}$ is the change in refractive index (RI) of the surrounding medium, which is usually caused by adsorbents; $d$ is the thickness of the binding layer, and $l$ is the plasmon effective decay length. According to the above equation, it is found that the plasmon response of nanoparticles decreased exponentially far away from the nanoparticle surface. Researchers have found that the enhanced electromagnetic fields in LSPR are strongly localized, with decay length of several nanometers in any direction normal to the nanoparticle surface. Further, the decay length of LSPR can be changed by tuning the nanoparticle size, shape, and composition [9-10]. Core-shell type is a new structure and the parameters of that can be tuned, such as Au shell, Au film and the diameter of Core and so on. So the core-shell nanosensor was studied in this paper. 


\section{Experiment section}

\subsection{LSPR detcetion system}

LSPR detection system was consisted of peristaltic pump, computer, flow-through cell, spectrograph, light source and so on. The solution provided by peristaltic pump flowed into the flow cell from the left beaker, and the water solution was collected in the right beaker shown in Figure 1(a). The input light produced by light source shined vertically the surface of the nanosensors by optical probe and the reflected signal was obtained by the spectrograph, and a computer processed the reflected signal to produce an output reflectivity or extinction spectrum. Figure 1(b) is a schematic diagram of core-shell nanosensors by the reflective mode. The core-shell nanosensor was fixed in flow-through cell. The reflectivity is equal to reflective light intensity divided by incident light intensity which is dimensionless number and is applied normalization method in this paper.
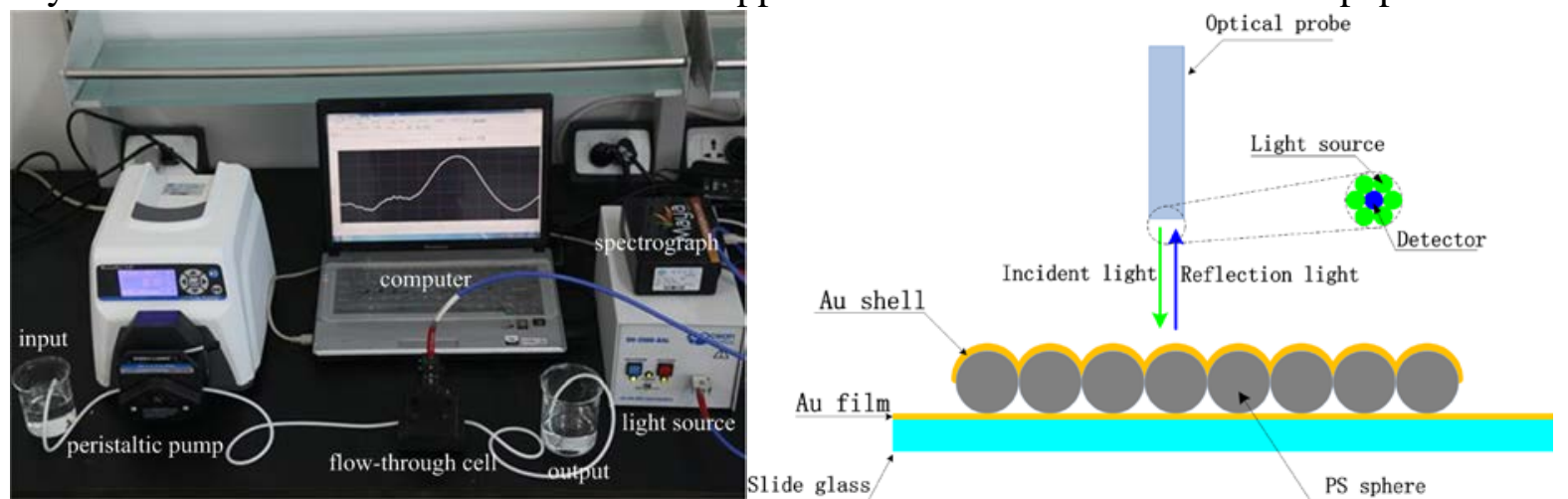

Figure 1. (a) A picture of the LSPR detection system (b) A schematic diagram of core-shell nanosensors using the reflective mode

\subsection{Preparation of core-shell nanosensors}

The core-shell nanosensor is layer substrate constructed by the monolayer closely-packed nanoparticle. The 590nm polysyrene nanosphere was employed as the "core" of the nanosensor, and a gold layer of 10nm was used as the "shell" of the nanosensor, which was deposited over the core surface. To form a steady and closely-packed monolayer, all glass slides were ultrasound in "piranha" solution $\left(\mathrm{H}_{2} \mathrm{O}_{2}: \mathrm{H}_{2} \mathrm{SO}_{4}, 3: 7\right.$ by volume) for 2 hours. And then, glass slides were put in ultrapure water and sonication in a mixed solution $\left(\mathrm{NH}_{3}: \mathrm{H}_{2} \mathrm{O}_{2}: \mathrm{H}_{2} \mathrm{O}=1: 1: 5\right.$ by volume) for 2 hours in order to get a hydrophilic surface. At last, all glass slides were cleaned in ultrapure water in which they were stored until be used. Next, a drop of mixture solution from a commercially purchased suspension of polystyrene spheres with a diameter of $590 \mathrm{~nm}$ at a concentration of about $20 \%$ and ethanol by volume 1:2 was spin-coated on the slide at a typical rotation rate of $3000 \mathrm{rpm}$ with $20 \mathrm{~s}$. The slide was slowly put into the container with ultrapure water. After the monolayer polysyrene kept stable in water, the monolayer polysyrene was absorbed on the other slide. Au shell and film were fabricated by using Ion Sputtering Instrument (Conditions: vacuum: $10 \mathrm{~Pa}$; current: $10 \mathrm{~mA}$; rate: $1 \mathrm{~nm} / \mathrm{s}$ ). The SEM image of the core-shell nanosensor was shown in Figure 2. It is found that nanoparticles are closely-packed each other, which offers an effective support for repeatability of the nanosensor in theory.

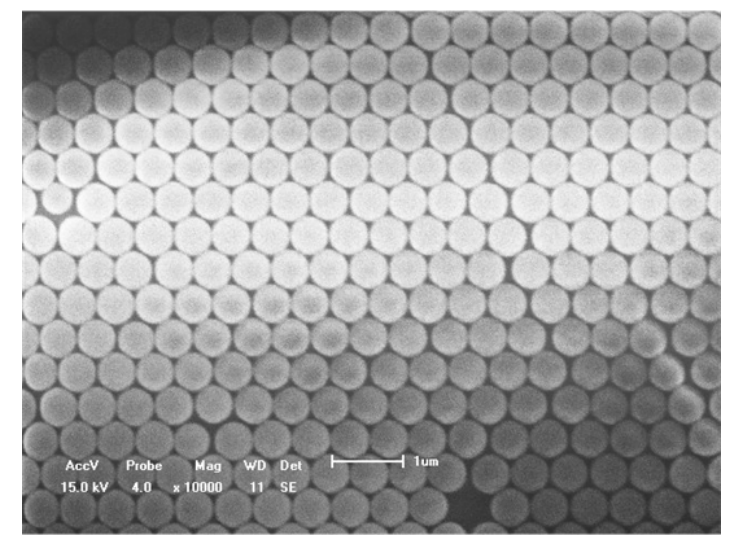

Figure 2 SEM image of the core-shell nanosensor 
3 The Optical Property of Core-shell Nanosensors

\subsection{The Measurement of Bulk Refractive Index Sensitivity}

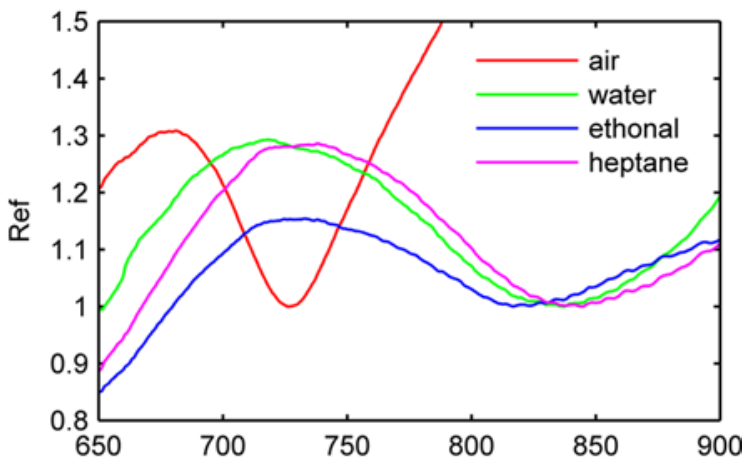

(a) wavelenth/nm

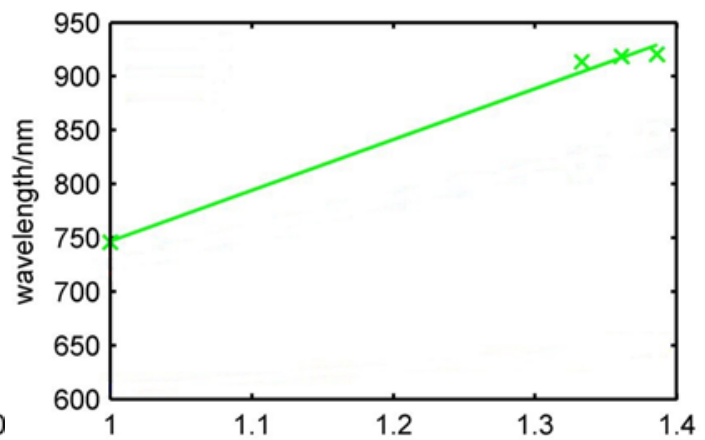

(b) refractive index

Figure 3. (a) The reflectivity value of the core-shell nanosensor in different solution with respect to air, water, ethanol and normal heptane, respectively. All values of the reflection are applied normalization method. (b) LSPR peaks of core-shell nanosensor in four mediums with respect to air, water, ethanol and normal heptane, respectively.

The simplest sensing application of the nanosensor is to detect changes in the bulk refractive index of their environment through shifts in the LSPR peak wavelength. It can be reported that the LSPR peak wavelength is approximately linear to changes in refractive index of the surrounding medium. Therefore, the refractive index sensitivity s of nanosensor is usually reported in nanometers of peak shift per refractive index unit (nm/RIU), that is

$$
s=d \lambda / d n
$$

As shown in Figure 5(b), although the Plasmon resonance wavelength is not strictly linear to the index of refraction, it is linear to a good approximation over small ranges of $n\left(R^{2}>0.95\right)$. From the linear regressions of Figure 3(b), we are able to calculate the bulk RIS (wavelength) s=470nm/RIU. It shows that the core-shell nanosensor of polystyrene is very sensitive to the surrounding medium, which can be useful for high sensitive detection.

\subsection{The measurement of The decay length}
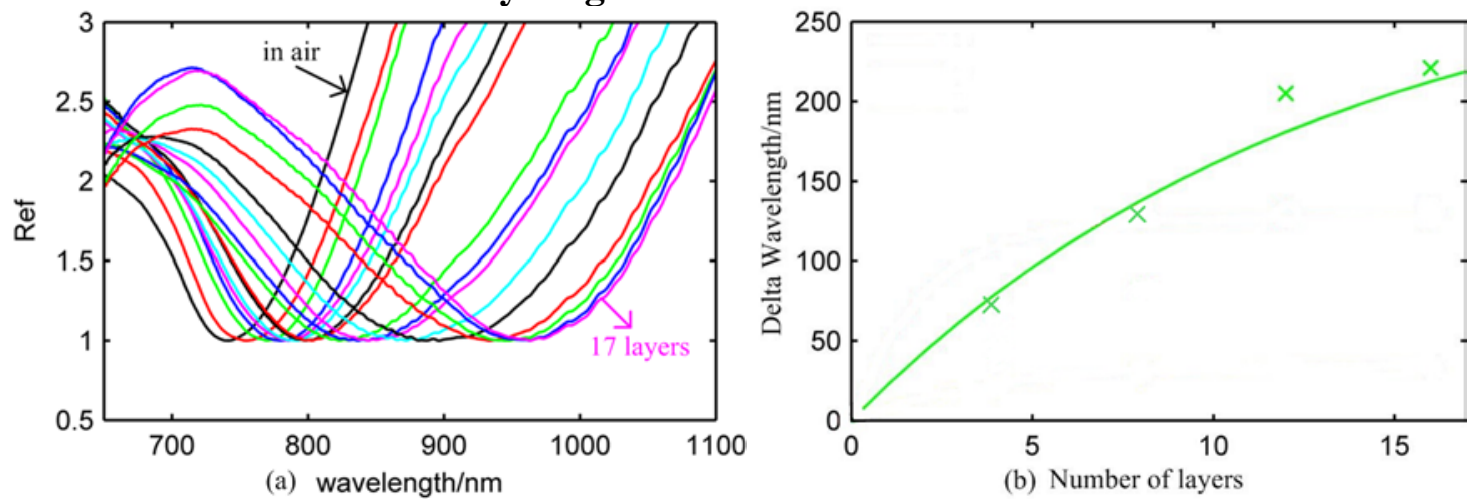

(b) Number of layers

Figure 4. (a) The plasmon resonant peak and reflectivity of the core-shell nanosensor in air. (b) The delta peak wavelength of the core-shell nanosensor as function of number of layers.

If the nanosensor was applied for biosensors, we must know the decay length to reach high sensitivity when binding event occurs. Because the field decay length of the nanosensor may be short, the sensitivity will decrease when the size of the biomolecule is out of that field. So the decay length is extremely important to measure the sensitivity of the nanosensor. Polyelectrolyte multilayers are simplicity, reproducibility and a highly controlled thickness. So Polyelectrolyte multilayers were employed to determine the decay length. Polyelectrolyte multilayers were deposited on the surface of Au shell and extinction spectra were measured after the binding of each bilayer. The LbL assembly was carried out until the plasmon peak wavelength and intensity were kept stable. The thickness of 
the layer was assumed to be $2.1 \mathrm{~nm}$ according to the measurement of the same multilayer system by Ofer Kedem [20].

Figure 4(a) is the reflectivity value of the core-shell nanosensor in air when the PAH/PSS bilayer was continuously absorbed on the surface of the core-shell nanosensor. The left black solid line is the reflectivity of the core-shell nanosensor. The right purple solid line is the reflectivity of the core-shell sensor which absorbed 17 layers PAH/PSS bilayer in air. The LSPR peaks shifts from $740 \mathrm{~nm}$ to 965 $\mathrm{nm}$ when the number of bilayers increase. The LSPR peak shows a stable tendency when the core-shell nanosensor absorbed 17 layers of the PAH/PSS bilayer and the LSPR wavelength peaks keep stable. For the core-shell nanosensor, four sets of data corresponding to the fourth, eighth, twelfth, sixteenth layer were selected to make an exponential curve fitting according to equation 1 shown in Figure 4(b). And then, the parameters can be obtained that $m=300 \mathrm{~nm} / \mathrm{RIU}, 1=26 \mathrm{~nm}$. The results show that this nanosensor is sensitive to surroundings around the surface of nanosensor. The decay length of this nanosensor shows that this naosensor is suitable for the small molecule detection and maybe obtain very high sensitivity.

\section{Summary}

We presented a systematic study on the fabrication and the Optical Property of Core-shell Nanosensor based on LSPR detection system. The core-shell nanosensor is fabricated by assembly and ion sputtering technology and shows good repeatability and stability. The RIS was measured by changing the surrounding medium in the refractive index. The decay length was obtained using polyelectrolyte LbL assembly method. Results show that this nanosensor can acquire high sensitivity and be used for small molecule detection.

\section{References}

[1] Hoa, X.D.; Kirk, A.G.; Tabrizian, M. Towards Integrated and Sensitive Surface plasmon Resonance Biosensors: a Review of Recent Progress. Biosens. Bioelectron 2007, 23, 151-160.

[2] Hirsch, L.R.; Jackson, J.B.; Lee, A.; Halas, N.J.; West, J.L. a Whole Blood Immunoassay Using Gold Nanoshells. Anal. Chem 2003, 75, 2377-2381.

[3] Haes, A.J.; Chang, L.; Klein, W.L.; Van Duyne, R.P. Detection of A Biomarker for Alzheimer's Disease from Synthetic and Clinical Samples Using a Nanoscale Optical Biosensor. J. Am. Chem. Soc 2005, 127, 2264-2271.

[4] Wu, T.F.; Yang, S.B.; Li, X.F. Tunable Plasmon Resonance Spectroscopy and Two-dimensional Anisotropy of Angular Optical Response of Overlapped Nanoshells. Opt. Express 2013, 21, 7811-7820.

[5] Willets, K.A.; Van Duyne, R.P. Localized Surface Plasmon Resonance Spectroscopy and Sensing. Annu. Rev. Phys. Chem 2007, 58, 267-297.

[6] Lance Kelly, K.; Coronado, E.; Zhao, L.L.; Schatz, G. The Optical Properties of Metal Nanoparticles: the Influence of Size, Shape and Dielectric Environment. J. Phys. Chem. B 2003, 107, 668-677.

[7] Himmelhaus, M.; Takei, H. Cap-shaped Gold Nanoparticles for an Optical Biosensor. Sens. Actuators B Chem 2000, 63, 24-30.

[8] Jung, L.S.; Campbell, C.T.; Chinowsky, T.M.; etc. Quantitative Interpretation of the Response of Surface Plasmon Resonance Sensors to Adsorbed Films. Langmuir 1998, 14, 5636-5648.

[9] Haes, A.J.; Zou, S.L.; Schatz, G.R.P. Van Duyne. A Nanoscale Optical Biosensor: the Long Range Distance Dependence of the Localized Surface Plasmon Resonance of Noble Metal Nanoparticles. J. Phys. Chem. B 2004, 108, 109-116. 
[10] Sannomiya, T.; Sahoo, P.; Mahcicek, D.; etc. Biosensing by Densely Packed and Optically Coupled Plasmonic Particle Arrays. J. Small 2009, 5, 1889-1896. 